\title{
Ein halbvolles oder halbleeres Glas?
}

\author{
Im Herbst letzten Jahres einigte sich die OECD auf die Einführung von Umwel- \\ trichtlinien für Exportkreditagenturen. Zum gleichen Zeitpunkt herrschte in \\ Deutschland Streit über eine Hermes-Bürgschaft für die Firma Siemens zur \\ Sicherung eines Turbinenverkaufs an ein finnisches Atomkraftwerk. Der Streit \\ unterstrich, wie groß der Reformbedarf von Exportrichtlinien war und auch \\ weiterhin ist. Denn letztendlich zeigt sich die Wirksamkeit der neven Richtlinien \\ erst bei Umsetzung in nationales Recht.
}

$\mathrm{I}$ m vergangenen Dezember kochte der Strei um eine eventuelle Exportförderung für $\mathrm{Zu}$ lieferungen der Firma Siemens zum Bau eines neuen finnischen Atomkraftwerkes hoch. Nachdem sich deutlich abzeichnete, dass Siemens trotz Fürsprache durch Kanzler und Wirtschaftsminister keine Hermes-Bürgschaft erhalten würde, zog der Konzern seine Anfrage zurück.

Trotzdem bleibt die Frage, wieso Siemens überhaupt einen Antrag auf Kreditbürgschaft gestellt hat. Der eigentliche Sinn von Hermes-Bürgschaften ist die Absicherung eines Exporteurs gegen Zahlungsausfall. Kann der Käufer seine Rechnungen nicht begleichen, übernimmt die Bundesregierung die durch den Ausfall entstandenen Kosten. Gleichzeitig sorgt sie meistens dafür, dass die Kosten in Staatsschulden des importierenden Landes gegenüber der BRD umgewandelt werden. Durch die Ausfallübernahme soll der Export in so genannte schwierige Märkte gefördert werden. Als schwierige Märkte gelten gemeinhin Schwellen- und Entwicklungsländer, in die deutsche Firmen sonst wegen des wirtschaftlichen Risikos nicht exportieren würden.

Dass der EU-Mitgliedsstaat Finnland nicht unter diese Kategorie fällt, ist offensichtlich. Die Gewährung von staatlichen Bürgschaften erleichtert aber die Finanzierung von Exportgeschäften durch kostengünstigere Kredite. Daher sind Hermes-Bürgschaften immer wieder als Subventionsinstrument genutzt und gescholten worden. Befürworter der uneingeschränkten Nutzung der Staatsbürgschaften führen an, dass jedes Industrieland und zunehmend auch Schwellenländer ähnliche Förderinstrumente zur generellen Unterstiutzung der heimischen Wirtschaft haben. Mit diesem Argument begründete auch Siemens seinen HermesAntrag. Die US-amerikanische Konkurrenz würde von der amerikanischen Export-Import-Bank unterstuitzt, weshalb für das eigene Angebot eine ähnliche Hilfe nötig sei. Letztendlich hat das deutsch-französische Konsortium den Auftrag auch ohne Hermes-Bürgschaft erhalten. Das Beispiel zeigt, dass Regelungen für Exportkreditagenturen zwar auf nationaler Ebene beginnen, jedoch international weiterdiskutiert und verabschiedet werden müssen.

Auf OECD-Ebene ist seit etwa acht Jahren über gemeinsame Umweltregeln für Exportkreditagenturen (ECA) diskutiert worden, die schließlich im Dezember 2003 verabschiedet worden sind.

\section{- Umweltvorreiter USA}

Auslöser der Neuregelung waren ausgerechnet die USA, die aufgrund von öffentlichem Druck bereits 1994 Umweltrichtlinien für ihre ECA, die Ex-ImBank, verabschiedet hatten. Die US-Umweltrichtlinien verlangen von heimischen Exporteuren die Einhaltung von Umweltstandards und die Bekanntmachung von Projekten vor der Entscheidung für eine Bürgschaft. Durch die Bekanntmachung werden interessierte und betroffene Kreise aufgefordert, ihre Bedenken gegenüber einem Projekt öffentlich zu bekunden. Genau diese beiden Punkte möchten die USA seitdem auch in internationalen Richtlinien verankert sehen.

Beide Punkte blieben auf OECD-Ebene jahrelang ein Zankapfel. 2001 kam es darüber sogar zum öffentlichen Eklat, als in einem auch ansonsten aus Umweltsicht wenig erfreulichen Abkommensentwurf speziell über die Punkte verbindliche Umweltstandards und Transparenz keine Einigung zwischen den USA und den übrigen OECD-Ländern erzielt werden konnte. Um nicht völlig unverbindliche Leitlinien gut zu heißen, stimmten die USA dem erarbeiteten Umweltabkommen gar nicht erst zu. Die übrigen Länder beschlossen Ab- kommen zunächst für zwei Jahre anzuwenden und dann eine Überprüfung durchzuführen, um zu einem für alle akzeptablen Ergebnis zu kommen.

Die Überprüfung fand im vergangenen Herbst statt. Bei den Verhandlungen ging es zum Beispiel darum, ab welchem Wert ein Projekt überhaupt geprüft werden muss. Ebenso sollte geklärt werden, wie Projekte nach ihren möglichen Umweltauswirkungen einzustufen sind. Die Kategorien reichen von A, ,schwerwiegende Umweltauswirkungen“ bis C „keine Auswirkungen zu erwarten“. In Kategorie A fallen etwa Großstaudämme, Atomkraftwerke, Pipelines oder Bergbausprojekte. Auch verhandelten die Vertragsparteien über die nötigen Umweltprüfungen, die nach einer Einstufung des Projekts zwingend durchgeführt werden müssen. Ob etwa nach Weltbankstandards auf mögliche Luft- oder Wasserverschmutzung geprüft wird und wieweit spezielle Schutzrichtlinien zu Naturschutzgebieten, zur Umsiedlung oder zu Indigenen angewandt werden. Gerade die speziellen Schutzrichtlinien machen Vorgaben, wie Konsultationen mit der von einem Projekt betroffenen Bevölkerung durchzuführen sind.

\section{Umsetzung offen}

Das Ergebnis der Verhandlungen lässt sich als halbvolles oder halbleeres Glas interpretieren: nach wie vor haben die verschiedenen ECAs die Wahl zwischen einer Reihe von Umweltstandards, bei denen auch solche regionaler Entwicklungsbanken herangezogen werden können, die zum Teil wenig ausgearbeitet oder verbindlich sind. Im Prinzip jedoch sollen die höheren Standards herangezogen werden. Soziale Fragen werden ebenfalls adressiert. Die Richtlinien verlangen, dass einige zentrale Schutzrichtlinien der Weltbank zum Beispiel zur Umsiedlung Anwendung finden sollen. Zwingend vorgeschrieben ist eine Umweltverträglichkeitsprüfung (UVP) bei besonders umweltrelevanten Projekten.

Da eine ordentlich durchgeführte UVP Konsultationen mit der betroffenen Bevölkerung voraussetzt, lässt sich daraus die Forderung nach Konsultation ableiten. Bei den besonders umweltrelevanten Projekten wird zudem zum ersten Mal verlangt, Umweltinformationen bereits vor der Entscheidung über die Gewährung von Bürgschaften zu veröffentlichen. Gerade im deutschen Kontext wäre die Übernahme dieser Anforderung ein echtes Novum, da bisher ex-ante Transparenz praktisch weder vorgesehen ist noch praktiziert wird. 
Gleichzeitig lassen die überarbeiteten Richtlinien eine Reihe Schlupflöcher offen, da im Prinzip alle Anforderungen zur Umweltprüfung unterlaufen werden können, solange dies in einem jährlichen Bericht dem OECD-Sekretariat angezeigt wird. Schlupflöcher dieser Art können natürlich das gesamte Abkommen ad absurdum führen.

Ob die neuen OECD-Richtlinien Umweltaspekte bei der Vergabe von Bürgschaften wirksam integrieren, wird sich erst bei der nationalen Umsetzungen der OECD-Vorgaben zeigen. Das hängt auch davon ab, wie hoch die Standards in den jeweiligen Ländern bereits sind. In Ländern, deren Exportkreditagenturen bereits einhalten müssen, was die OECD-Leitlinien vorschreiben, oder sogar darüber hinausgehen, befürchten Umweltorganisationen einen Abwärtstrend in ökologischen und sozialen Fragen.

\section{Zieht Deutschland nach?}

In Deutschland hingegen ergeben sich aus den neuen OECD-Leitlinien neue Ansätze. Alle skizzierten Anforderungen an Umweltprüfungen sind bisher bei der Vergabe von Hermes-Bürgschaften nicht vorgesehen und bedürfen der Anpassung in den Hermes-Leitlinien. Die Bundesregierung ist in der Verantwortung, die internationalen Vorgaben umzusetzen. Dabei kann sie durch eine engagierte Interpretation eine Vorreiterrolle übernehmen und durch hohe Standards beweisen, dass der befürchtete Abwärtstrend nicht stattfindet und sich die positiven Aspekte der OECD-Leitlinien verbindlich durchsetzen. Bisher hat sich die Bundesregierung bei den Exportkreditagenturen international vor allem als Bremser hervorgetan und auch jetzt betont das Wirtschaftsministerium bereits, dass sich aus dem neuen Abkommen kein Anpassungsbedarf ergebe.

Es ist jedoch die Legislative gefragt, die Interpretation nicht der Exekutiven zu überlassen, sondern die Chance zu nutzen, die sich aus den internationalen Neuerungen ergibt. So kann sie den Versprechungen aus dem Koalitionsvertrag nachkommen, im Sinne einer nachhaltigen Entwicklung die Vergabe von Hermes-Bürgschaften transparenter zu gestalten und Weltbankstandards einzuhalten. Bei der Gelegenheit könnte sie auch ein entscheidendes Schlupfloch stopfen, das der Streit um die Bürgschaft für das finnische Atomkraftwerk deutlich gezeigt hat. In einem einzigen Punkt sind nämlich die Hermes-Leitlinien ähnlichen internationalen Vorschriften voraus: sie schließen die Exportförderung von Nukleartechnologie aus. Bereits bei der Verabschiedung der Hermes-Leitlinien im April 2001 haben Umweltorganisationen gewarnt, dass die Formulierung Interpretationsspielräume ließe. Genau diese wollten Siemens und das Wirtschaftsministerium im Fall Finnland nutzen. Beim zu verbürgenden Export handelte es sich um Turbinen und die, so das Argument, seien ja schließlich keine Nukleartechnologie. Da Siemens den Antrag zurückgezogen hat, wurde kein Präzedenzfall über die nähere Bestimmung von Nukleartechnologien in diesem Zusammenhang geschaffen. Deutlich geworden ist jedoch, dass sich Anpassungsbedarf nicht nur aus den OECD-Leitlinien ergibt, sondern auch das Atom-Ausschlusskriterium präzisiert werden muss, wenn es tatsächlich seine Bestimmung erfuillen soll.

\section{Die Autorin}

Regine Richter ist Mitarbeiterin bei der Umwelt- und Menschenrechtsorganisation urgewald.

Kontakt: urgewald e.V., Im Grünen Haus, Prenzlauer Allee 230, 10405 Berlin. Tel. 030-44339169,

E-Mail: regine@urgewald.de

\section{Ökologisches Wirtschaften Sparen Sie sich Zeit und Geld mit dem $\mathrm{Abo}^{+}$!}

_Sie teilen sich Ökologisches Wirtschaften mit den anderen 20 Kollegen im Verteiler und bekommen sie erst Monate später in die Hand?

_Sie verbringen einige Zeit im Ausland, wollen aber keine Ausgabe von Ökologisches Wirtschaften verpassen und es pünktlich und kostengünstig erhalten?

Das $\mathrm{Abo}^{+}$von Ökologisches Wirtschaften kann Ihre Probleme lösen:

_Bestellen Sie zu Ihrem Print-Abo zusätzlich ein Online-Abo zum halben Preis!

_Sie erhalten die Online-Version eine Woche vor dem Erscheinen der Print-Version.

_Schluss mit der Warteschlange am Kopierer, drucken Sie sich die interessanten Artikel

bequem an Ihrem Rechner aus!

*) Abo ${ }^{+}$funktioniert nur in Kombination mit einem Print-Abo von Ökologisches Wirtschaften.

Firma, Abteilung

Name, Vorname:

Straße, Postfach:

PLZ/Ort:

E-Mail:

Kundennr:
$\mathrm{Ja}$, ich bestelle $\mathrm{Abo}^{+}$(Print- und Online-Abo) als

Firmenabo für $142,50 €$

Normalabo für $72,00 €$

Studentenabo für $48,00 €$

Ja, ich bestelle die Online-Version zusätzlich

$\bigcirc$ für Firmen zum Aufpreis von 47,50€

$\bigcirc$ als Normalabonnent zum Aufpreis von 24,00€

für Studenten zum Aufpreis von 16,00€ 
(c) 20I0 Authors; licensee IÖW and oekom verlag. This is an article distributed under the terms of the Creative Commons Attribution Non-Commercial No Derivates License (http://creativecommons.org/licenses/by-nc-nd/3.o/), which permits unrestricted use, distribution, and reproduction in any medium, provided the original work is properly cited. 